\title{
De novo entecavir+adefovir
} CrossMark dipivoxil+lamivudine triple-resistance mutations resulting from sequential therapy with adefovir dipivoxil, and lamivudine

\author{
Song Yang ${ }^{1}$, Huichun Xing ${ }^{1}$, Qi Wang ${ }^{1}$, Xiaomei Wang ${ }^{1}$, Shunai Liu² and Jun Cheng ${ }^{1,2^{*}}$
}

\begin{abstract}
Background: Entecavir-resistance mutations are commonly induced by entecavir treatment in chronic hepatitis B patients. However, entecavir+adefovir dipivoxil+lamivudine triple-resistance mutations induced by sequential or combination treatment with lamivudine and adefovir dipivoxil have never been reported.

Results: We retrospectively reviewed 1200 patients who had been tested for anti-HBV drug resistance at Beijing Ditan Hospital of Capital Medical University, and five patients showing multidrug resistance to lamivudine and adefovir dipivoxil were enrolled. Stored serum samples were used for genetic analysis, which yielded a total of 135 clones. Entecavir+adefovir dipivoxil+lamivudine triple-resistance mutations were identified in 60 \% (3/5) entecavirnaïve patients who received sequential therapy with adefovir dipivoxil and lamivudine. Specifically, we found one rtM204I+rtL180 M+rtM250 V+rtA181 V clone among 23 clones from patient 1 (4.35 \%), one rtM204 V+vrtL180 M +rtM250 V+rtA181 V clone among 24 clones from patient 2 (4.17\%), and 2 clones harboring rtM204 V+rtL180 M+ rtM250 V+rtA181 V and rtM204 V+rtL180 M+rtl169 V+rtA181 V among 20 clones from patient 3 (10.0 \%). The other 2 patients showed multidrug resistance after lamivudine/telbivudine and adefovir dipivoxil combination therapy, but no entecavir-resistance mutations were found in these two patients.
\end{abstract}

Conclusion: De novo entecavir+adefovir dipivoxil+lamivudine triple-resistance mutations can be induced by sequential therapy with adefovir dipivoxil and lamivudine in patients who never take entecavir. These results provide important information for sequential therapy with adefovir dipivoxil and lamivudine and the use of entecavir as a rescue therapy for these patients with multidrug resistance.

Keywords: Hepatitis B virus, Multidrug resistance, Entecavir, Adefovir dipivoxil, Lamivudine

\section{Findings}

Chronic hepatitis B virus (HBV) infection is estimated to affect approximately 120 million people in China, with an annual death toll of approximately 300,000, mostly resulting from $\mathrm{HBV}$-related cirrhosis and hepatocellular carcinoma (HCC) [1]. Although antiviral therapy with nucleoside/nucleotide analogues (NAs) and interferons (IFNs) can be used to suppress HBV replication

\footnotetext{
*Correspondence: chengj0817@sina.cn

${ }^{1}$ Center of Hepatology, Beijing Ditan Hospital, Capital Medical University,

8 East Jingshun Street, Chaoyang District, Beijing 100015, China

Full list of author information is available at the end of the article
}

and prevent disease progression, the side effects of antiviral drugs should be monitored regularly [2, 3]. Five NAs are currently approved for anti-HBV treatment in China, including lamivudine (LAM), adefovir dipivoxil (ADV), telbivudine (LdT), entecavir (ETV), and tenofovir disoproxil fumarate (TDF). One major concern for NA therapies is the emergence of HBV resistance after prolonged treatment, especially for drugs with low resistance barriers (LAM, ADV, and LdT) [2, 3]. In some patients, mutations causing multidrug resistance (MDR) may occur after sequential monotherapy with low-resistance barrier NAs [3]. Yim et al. reported three 
cases of LAM+ADV-resistant patients after sequential therapy with LAM and ADV [4]. Liu et al. detected a ETV+ADV+LAM triple-resistant $\mathrm{HBV}$ strain in a LAM+ADV-resistant patient who had taken sequential ETV as a rescue therapy [5]. Additionally, Kim et al. reported six cases of ETV+ADV+LAM resistance that occurred after sequential therapy with LAM, ADV, and ETV [6].

ETV resistance mutations are commonly induced by ETV treatment. However, Yang et al. reported that LAM-resistant HBV also had reduced susceptibility to ETV (37- to 471-fold reduction) [7]. Additionally, Inoue et al. described a patient who received LAM+ADV and then developed de novo ETV resistance with rtM204 V+rtL180 M+rtT184 S mutations [8]. However, it is still not clear whether or not sequential or combination therapy with LAM and ADV induces ETV+ADV+LAM triple-resistance mutations. Furthermore, ETV+ADV is still used for rescue therapy in many LAM+ADV-resistant patients [9-11], especially in China where TDF is costly and not covered by the reimbursement system. Therefore, we retrospectively analyzed chronic hepatitis B (CHB) patients in Beijing Ditan Hospital of Capital Medical University in whom sequential or combination therapy with LAM+ADV was not successful. Genetic analyses of stored serum samples revealed ETV+ADV+LAM triple-resistance mutations in three ETV-naïve patients who received sequential therapy with ADV+LAM.

The initial study population consisted of $1200 \mathrm{CHB}$ patients who underwent drug resistance testing by nested polymerase chain reaction (PCR)-based direct sequencing at the Beijing Ditan Hospital. Written consent was obtained from each patient. Demographic and clinical data were collected using a questionnaire. Serum samples were stored at $-20{ }^{\circ} \mathrm{C}$ until further analysis. Patients exhibiting MDR to both LAM and ADV were selected for the study. Clinical data were confirmed by checking medical records in the hospital information system. This study was approved by ethics committee of Beijing Ditan Hospital.

HBV DNA extraction was conducted using an AxyPrep Body Fluid Viral DNA/RNA Miniprep Kit (Corning Inc., Corning, NY). Nested PCR-direct sequencing was performed as previously described [12]. The PCR products were purified using a QIAquick PCR purification Kit (Qiagen, Valencia, CA, USA) according to the manufacturer's instructions. Purified DNA was sequenced using an automated ABI 3730 DNA sequencer (Applied Biosystems, Foster City, CA, USA) at Beijing Augct Bioengineering Co., Ltd. DNA sequences were aligned using SeqMan and EditSeq software (DNASTAR Inc., Madison, WI). The HBV polymerase sequencing results were also used for HBV genotyping with the genotyping tool of the website of the National Center for Biotechnology Information [13]. PCR-amplified HBV DNA was cloned into the pGEM-T easy vector (Promega, Madison, WI) according to the manufacturer's instructions. Clones $(n=20-38)$ were selected from each patient, and the sequences were analyzed using MegAlign software (DNASTAR Inc.).

Five HBV patients with MDR to both LAM/LdT and ADV were enrolled. The demographic and clinical characteristics of the patients, together with the direct sequencing results of the nested PCR products, are shown in Table 1. HBV genotyping showed that all 5 patients were infected with genotype $\mathrm{C} \mathrm{HBV}$.

In total, 135 clones were obtained from the five patients. All clones were submitted to NCBI GenBank, and the accession numbers are shown in Additional file 1: Table S1. The results of the clonal analysis are shown in Fig. 1. Patient 1 received ADV and LAM sequential therapy, and direct sequencing of the nested PCR product showed rtM204 V+rtL180 M+rtA181 V mutations upon secondary virological breakthrough, whereas rtM204 V+rtL180 M+rtA181 V strains accounted for $78.26 \%(18 / 23)$ of the clones in the clonal analysis. In addition, $17.39 \%(4 / 23)$ and $13.04 \%(3 / 23)$ of the clones harbored the rtM204 I and rtA181 T mutations, respectively, which were not found by direct sequencing. Moreover, one clone (4.35 \%) harbored rtM204 I+rtL180 M+rtM250 V+rtA181 V mutations, which resulted in resistance to LAM+ADV+ETV (Additional file 2: Figure S1); however, this patient had never received ETV therapy.

Patient 2 received ADV and LAM sequential therapy, and sequencing revealed rtM204 V+rtL180 M+rtA181 V mutations upon secondary virological breakthrough, whereas rtM204 V+rtL180 M+rtA181 V strains comprised $95.83 \%(23 / 24)$ of the clones in the clonal analysis. Additionally, one clone (4.17 \%) bore rtM204 V+rtL180 $\mathrm{M}+\mathrm{rtM} 250 \mathrm{~V}+\mathrm{rtA} 181 \mathrm{~V}$ mutations, which resulted in resistance to LAM+ADV+ETV (Additional file 3: Figure S2). This patient also never received ETV therapy.

Patient 3 received ADV and LAM sequential therapy, and sequencing showed rtM204 V+rtL180 M+rtA181 V mutations upon secondary virological breakthrough, whereas $85.0 \%(17 / 20)$ of the clones harbored rtM204 V+rtL180 M+rtA181 V mutations. In addition, one clone $(5.0 \%)$ bore rtM204 $\mathrm{V}+\mathrm{rtA} 181 \mathrm{~T}$ mutations. Moreover, two clones (10.0 \%) bore ETV+LAM+ADV triple-resistance mutations (Additional file 4: Figure S3 and Additional file 5: Figure S4), even though this patient had also never received ETV therapy.

Patient 4 received ADV+LAM for rescue therapy upon ADV resistance, and sequencing showed rtM204 
Table 1 Demographic and clinical characteristics of patients

\begin{tabular}{|c|c|c|c|c|c|}
\hline Patient & 1 & 2 & 3 & 4 & 5 \\
\hline Age (years) & 61 & 36 & 55 & 38 & 40 \\
\hline Sex & $\mathrm{F}$ & M & M & M & M \\
\hline Race & Asian & Asian & Asian & Asian & Asian \\
\hline Diagnosis & $\mathrm{CHB}$ & $\mathrm{CHB}$ & $\begin{array}{l}\text { HBV-related } \\
\text { cirrhosis, HCC }\end{array}$ & $\mathrm{CHB}$ & $\mathrm{CHB}$ \\
\hline HBV genotype & C & $C$ & $\mathrm{C}$ & C & $C$ \\
\hline HBeAg/Anti-HBe & $-/+$ & $+/-$ & $+/-$ & $+/-$ & $+/-$ \\
\hline $\begin{array}{l}\text { Antiviral treatment history } \\
\text { (months) }\end{array}$ & $\begin{array}{l}\text { ADV (36) } \\
\downarrow^{a} \\
\text { LAM (12) }\end{array}$ & $\begin{array}{l}\text { ADV (36) } \\
\downarrow \\
\operatorname{LAM}(12)\end{array}$ & $\begin{array}{l}\text { ADV (12) } \\
\downarrow \\
\operatorname{LAM}(12)\end{array}$ & $\begin{array}{l}\text { ADV (12) } \\
\downarrow \\
\text { LAM+ADV (8) }\end{array}$ & $\begin{array}{l}\text { LAM (36) } \\
\downarrow \\
\text { LAM+ADV (8) } \\
\downarrow \\
\text { LdT (6) }\end{array}$ \\
\hline Results of sequencing & $\begin{array}{l}\text { rtM204V+rtL180 M } \\
+ \text { rtA181 V }\end{array}$ & $\begin{array}{l}\text { rtM204V+rtL180 M } \\
+ \text { rtA181 V }\end{array}$ & $\begin{array}{l}\text { rtM204 V } \\
\quad+\text { rtL180 M } \\
\quad+\text { rtA181 V }\end{array}$ & $\begin{array}{l}\text { rtM204 I+rtA } \\
181 \mathrm{~V} / \mathrm{T}\end{array}$ & $\begin{array}{l}\text { rtM204 V+rtL180 M+ } \\
\text { rtA181 V }\end{array}$ \\
\hline
\end{tabular}

$A D V$ adefovir dipivoxil, $L A M$ lamivudine, $L d T$ telbivudine, $P C R$ polymerase chain reaction, $C H B$ chronic hepatitis $B, H C C$ hepatocellular carcinoma, $L A M+A D V L A M$ and ADV combination therapy

a $\downarrow$ indicates followed by

I+rtA181 V+rtA181 T mutations upon secondary virological breakthrough. A high diversity of mutated strains was observed in the clonal analysis, with seven mutation patterns present in 30 clones. The rtN236 T mutation was not found by direct sequencing of PCR products, and no ETV-resistance mutations were detected in this patient.

Patient 5 received ADV+LAM for rescue therapy upon LAM resistance, but was later switched to LdT monotherapy, which was requested by the patient because of concerns regarding creatinine elevation. Sequencing identified rtM204 V+rtL180 M+rtA181 V mutations upon secondary virological breakthrough. All clones harbored rtM204 V+rtL180 M+rtA181 V mutations, consistent with the sequencing results, and no de novo ETV resistance mutations were detected.

The possible overlapping S-gene mutations of all clones harboring the rtA181 T mutation were analyzed (Additional file 6: Table S2). In patient 1, 3 clones harboring the rtA181 T mutation had sW172*(stop codon) mutations in the overlapping $\mathrm{S}$ gene. Additionally, 1 clone and 15 clones harboring rtA181 T mutations in in patients 3 and 4 , respectively, showed sW172* mutations.

In this study, we performed a genetic analysis with serum samples obtained from five patients with chronic HBV infection that exhibited MDR to LAM/LdT and ADV following sequential/combination therapy with LAM/LdT and ADV. Surprisingly, de novo ETV resistance mutations were present in all three patients who received sequential therapy with ADV+LAM. Inoue et al. previously performed a clonal analysis and reported that strains with ETV resistance harbored only ETV resistance mutations and no ADV resistance mutations.
However, in the present study, all three patients had HBV strains with MDR for ETV+LAM+ADV, although these MDR clones comprised only a minor population of the quasispecies.

There are several reports of the use of ETV as a rescue therapy for patients with MDR to LAM+ADV. However, Heo et al. reported that ETV monotherapy is inferior as a rescue therapy in patients with MDR to LAM and ADV [14]. Additionally, Xu et al. reported the use of ETV+ADV for rescue therapy in 45 patients who failed to respond to treatment with multiple NAs, with $2 / 45$ patients showing LAM+ADV resistance at baseline. However, after 24 months of treatment with ETV+ADV treatment, one patient still did not achieve a complete virological response (HBV DNA $\leq 500$ copies/mL) (11). Moreover, Lim et al. reported that ETV + ADV could be used as a rescue therapy in patients with a suboptimal response to LAM+ADV; however, only $28.9 \%(13 / 45)$ of the patients achieved virological responses (HBV DNA $<60 \mathrm{IU} / \mathrm{mL}$ ) after 52 months of therapy [15]. Notably, this study also included two patients with de novo ETV resistance mutations at rtT184 A and rtM250 L after LAM+ADV treatment, but no further clonal analyses were conducted in these patients. According to these studies, de novo ETV-resistance mutations after sequential and/or combination therapy with LAM+ADV may play a role in the inferior efficacy of ETV rescue therapy in these patients.

The present study also indicates a need for more sensitive HBV drug resistance tests in clinical practice. Direct sequencing of PCR products is frequently used in clinical practice and trials to detect NA resistance $[16,17]$; 
patient 1

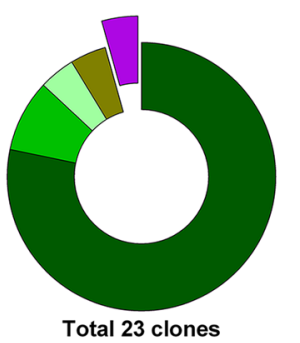

- rtM204V+rtL180M+rtA181V 18 clones $(78.26 \%)$

$\square$ rtM204I+rtA181T 2 clones $(8.70 \%)$

$\square$ rtM204V+rtA181T 1 clone $(4.35 \%)$

$\square$ rtM204I+rtL180M+rtA181V 1 clone (4.35\%)

- rtM204V+rtL180M+rtM250V+rtA181V 1 clone $(4.35 \%)$

patient 2

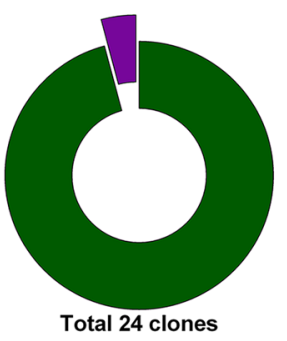

rtM204V+rtL180M+rtA181V 23 clones $(95.83 \%)$

- rtM204V+rtL180M+rtM250V+rtA181V 1 clone (4.17\%)

\section{patient 3}

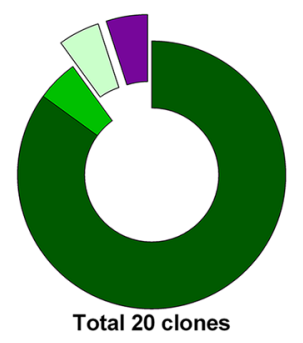

rtM204V+rtL180M+rtA181V 17 clones(85.0\%)

rtM204V+rtA181T 1 clones $(5.0 \%)$

rtM204V+rtL 180M+rtM250V+rtA181V 1 clone $(5.0 \%)$

rtM204V+rtL180M+rtl169V+rtA181V 1 clone $(5.0 \%)$

\section{patient 4}
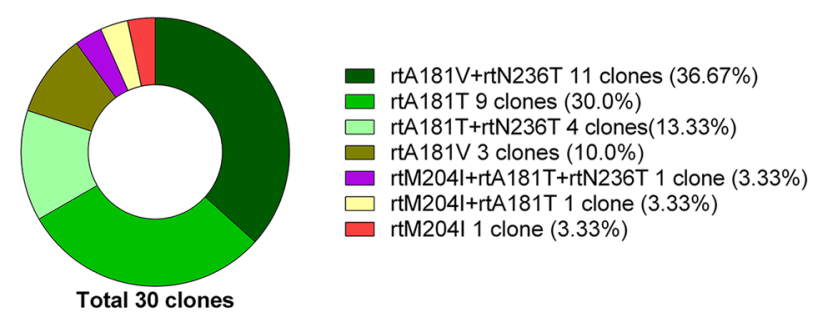

\section{patient 5}

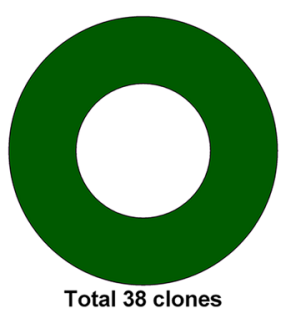

rtM204V+rtL180M+rtA181V 38 clones (100.0\%)

Fig. 1 Genetic analysis of multidrug-resistant strains (135 clones) from patients receiving nucleoside/nucleotide analogue treatment. The expanded sections show de novo entecavir resistance mutations 
however, it can only identify mutations when they reach approximately $20 \%$ of the total HBV quasispecies pool [18]. As shown in this study, direct sequencing of PCR products was unable to detect de novo ETV-resistant strains in patients 1,2 , and 3 , as these strains comprised $4.35,4.17$, and $15.0 \%$ of the total quasispecies, respectively. However, minor mutant strains can evolve to major strains under ETV selection pressure, and thus their early detection is important. More sensitive resistance tests have been reported to detect mutant strains comprising $<5 \%$ of the total HBV quasispecies, but they are either costly or inconvenient for widespread use in China $[19,20]$.

The study also calls attention to the use of less potent NAs in combination as a rescue therapy for NA resistance [2,3]. Addition of LAM is a therapeutic option for ADV-resistant patients. In this study, patient 4 developed MDR to LAM+ADV while receiving LAM+ADV combination therapy to rescue ADV resistance. Other reports have described similar phenomena $[8,15]$. Thus, thorough and rapid inhibition of HBV replication is critical for combination therapy; otherwise, combination therapy with LAM+ADV may increase the selection pressure of both drugs in patients with persistent viremia.

In this study, all MDR patients were infected with genotype $\mathrm{C}$. we consider this is partly because genotype $\mathrm{C}$ is predominant in the northern part of China [1]. Several reports have indicated higher numbers of ADV or LAM resistance mutations in HBV genotype $C$ compared with genotype $B[21,22]$. However, the sample size was too small to establish a correlation between genotype $\mathrm{C}$ and MDR mutations.

It has been reported that the rtA181 $\mathrm{T}$ mutation in HBV may cause sW172* in the overlapping $S$ gene in genotype D HBV infection [23]. In this study, all genotype $\mathrm{C}$ HBV clones harboring the rtA181 $\mathrm{T}$ mutation had sW172* mutations in the overlapping S-gene. Strains harboring the rtA181 $\mathrm{T} / \mathrm{sW} 172^{*}$ mutation accounted for $13.04 \%$ (3/23), $4.17 \%(1 / 24)$, and $50.0 \%(15 / 30)$ of the total clones in patients 1,3 , and 4 , respectively. This also confirms that the HBV rtA181 T/sW172"mutation is usually detected in a mixed population with lower replication efficiency [24].

In conclusion, our results indicate that de novo $\mathrm{LAM}+\mathrm{ADV}+\mathrm{ETV}$ resistance mutations may be induced by sequential therapy with ADV+LAM in patients who never took ETV. These results provide important information for administration of sequential therapy with ADV and LAM and for the use of ETV for rescue therapy in patients with MDR to ADV+LAM.

\section{Additional files}

Additional file 1: Table S1. GenBank accession numbers of 135 sequences from 5 chronic hepatitis B patients.

Additional file 2: Figure S1. Electropherogram of rtM204 V+rtL180 M+rtA181 V+rtM250 V clone in Patient 1 (GenBank accession number: KU736795).

Additional file 3: Figure S2. Electropherogram of rtM204 V+rtL180 M+rtA181 V+rtM250 V clone in Patient 2 (GenBank accession number: KU751680).

Additional file 4: Figure S3. Electropherogram of rtM204 V+rtL180 $\mathrm{M}+\mathrm{rtA} 181 \mathrm{~V}+\mathrm{rtl} 169 \mathrm{~V}$ clone in Patient 3 (GenBank accession number: KU751729).

Additional file 5: Figure S4. Electropherogram of rtM204 V+rtL180 M+rtA181 V+rtM250 V clone in Patient 3 (GenBank accession number: KU751733).

Additional file 6: Table S2. Overlapping HBsAg mutations caused by HBV rtA181 T mutation.

\section{Abbreviations}

HBV: hepatitis B virus; MDR: multidrug resistance; LAM: lamivudine; ADV: adefovir dipivoxil; LdT: telbivudine; ETV: entecavir; TDF: tenofovir disoproxil fumarate; NA: nucleoside/nucleotide analogue.

\section{Authors' contributions}

CJ contributed to the study design, critical review of the manuscript, and approval of the final draft. YS contributed to data collection, lab testing, and drafting of the manuscript. WQ, WX, and LS contributed to data collection and lab testing. XH contributed to study design and critical review of the manuscript. All authors read and approved the final manuscript.

\section{Author details}

${ }^{1}$ Center of Hepatology, Beijing Ditan Hospital, Capital Medical University, 8 East Jingshun Street, Chaoyang District, Beijing 100015, China. ${ }^{2}$ Institute of Infectious Diseases, Beijing Ditan Hospital, Capital Medical University, 8 East Jingshun Street, Chaoyang District, Beijing 100015, China.

\section{Acknowledgements}

The study was supported by Beijing Municipal Administration of Hospitals Clinical Medicine Development of Special Funding Support (No. ZY201402) and the Capital Health Research and Development of Special (No. 2011-2017-02).

\section{Competing interests}

All authors declare that they have no competing interests.

Received: 5 November 2015 Accepted: 29 March 2016

Published online: 14 April 2016

References

1. Cui Y, Jia J. Update on epidemiology of hepatitis B and C in China. J Gastroenterol Hepatol. 2013;28(Suppl 1):7-10.

2. EASL clinical practice guidelines. Management of chronic hepatitis $B$ virus infection. J Hepatol. 2012;57:167-85.

3. Zoulim F, Locarnini S. Hepatitis B virus resistance to nucleos(t)ide analogues. Gastroenterology. 2009;137:1593-608.

4. Yim HJ, Hussain M, Liu Y, Wong SN, Fung SK, Lok AS. Evolution of multidrug resistant hepatitis B virus during sequential therapy. Hepatology. 2006;44:703-12. 
5. Liu Y, Wang C, Zhong Y, Chen L, Li X, Ji D, et al. Evolution and suppression of HBV strains with multidrug resistance to lamivudine, adefovir dipivoxil and entecavir in a patient with chronic hepatitis B. Antivir Ther. 2010;15:1185-90.

6. Kim SS, Cho SW, Kim SO, Hong SP, Cheong JY. Multidrug-resistant hepatitis B virus resulting from sequential monotherapy with lamivudine, adefovir, and entecavir: clonal evolution during lamivudine plus adefovir therapy. J Med Virol. 2013;85:55-64.

7. Yang H, Qi X, Sabogal A, Miller M, Xiong S, Delaney WE. Cross-resistance testing of next-generation nucleoside and nucleotide analogues against lamivudine-resistant HBV. Antivir Ther. 2005:10:625-33.

8. Inoue J, Ueno Y, Wakui Y, Niitsuma H, Fukushima K, Yamagiwa Y, et al. Four-year study of lamivudine and adefovir combination therapy in lamivudine-resistant hepatitis B patients: influence of hepatitis B virus genotype and resistance mutation pattern. J Viral Hepat. 2011;18:206-15.

9. Park MS, Kim BK, Kim KS, Kim JK, Kim SU, Park JY, et al. Antiviral efficacies of currently available rescue therapies for multidrug-resistant chronic hepatitis B. Clin Mol Hepatol. 2013;19:29-35.

10. Chon YE, Jin B, Ahn SH, Kim S, Kim ND, Park JH, et al. Clonal evolution of multidrug resistant hepatitis $B$ virus during entecavir rescue therapy. Liver Int. 2015;35:2370-83.

11. Xu XH, Li GL, Qin Y, Li Q, He FQ, Li JY, et al. Entecavir plus adefovir rescue therapy for chronic hepatitis B patients after multiple treatment failures in real-life practice. Virol J. 2013;10:162.

12. Yan J, Xie W, Wang Q, Li Y, Feng X, Cheng J. The optimal threshold: baseline serum hepatitis B virus DNA and alanine transaminase levels can predict the 2-year on-treatment virological response to lamivudine. Hepat Mon. 2011;11:358-63.

13. Rozanov M, Plikat U, Chappey C, Kochergin A, Tatusova T. A webbased genotyping resource for viral sequences. Nucleic Acids Res. 2004;32:W654-9.

14. Heo NY, Lim YS, Lee HC, Chung YH, Lee YS, Suh DJ. Lamivudine plus adefovir or entecavir for patients with chronic hepatitis B resistant to lamivudine and adefovir. J Hepatol. 2010;53:449-54.

15. Lim YS, Lee JY, Lee D, Shim JH, Lee HC, Lee YS, et al. Randomized trial of entecavir plus adefovir in patients with lamivudine-resistant chronic hepatitis B who show suboptimal response to lamivudine plus adefovir. Antimicrob Agents Chemother. 2012;56:2941-7.
16. Marcellin P, Heathcote EJ, Buti M, Gane E, de Man RA, Krastev Z, et al. Tenofovir disoproxil fumarate versus adefovir dipivoxil for chronic hepatitis B. N Engl J Med. 2008;359:2442-55.

17. Lai CL, Shouval D, Lok AS, Chang TT, Cheinquer H, Goodman Z, et al. Entecavir versus lamivudine for patients with $\mathrm{HBeAg}$-negative chronic hepatitis B. N Engl J Med. 2006;354:1011-20.

18. Lok AS, Zoulim F, Locarnini S, Bartholomeusz A, Ghany MG, Pawlotsky $J M$, et al. Antiviral drug-resistant HBV: standardization of nomenclature and assays and recommendations for management. Hepatology. 2007;46:254-65.

19. Niesters HG, Zoulim F, Pichoud C, Buti M, Shapiro F, D'Heuvaert N, et al. Validation of the INNO-LiPA HBV DR assay (version 2) in monitoring hepatitis $B$ virus-infected patients receiving nucleoside analog treatment. Antimicrob Agents Chemother. 2010;54:1283-9.

20. Kim HS, Han KH, Ahn SH, Kim EO, Chang HY, Moon MS, et al. Evaluation of methods for monitoring drug resistance in chronic hepatitis B patients during lamivudine therapy based on mass spectrometry and reverse hybridization. Antivir Ther. 2005;10:441-9.

21. Li W, Warner N, Sozzi V, Yuen L, Colledge D, Li T, et al. Hepatitis B virus genotype $C$ encoding resistance mutations that emerge during adefovir dipivoxil therapy: in vitro replication phenotype. Hepatol Int 2013;7:443-50.

22. Li X, Wang L, Zhong Y, Wong VW, Xu Z, Liu Y, et al. Hepatitis B virus (HBV) subgenotypes $C 2$ and B2 differ in lamivudine- and adefovir-resistanceassociated mutational patterns in HBV-infected Chinese patients. J Clin Microbiol. 2010;48:4363-9.

23. Warner N, Locarnini S. The antiviral drug selected hepatitis B virus rtA181T/sW172* mutant has a dominant negative secretion defect and alters the typical profile of viral rebound. Hepatology. 2008;48:88-98.

24. Ahn SH, Park YK, Park ES, Kim JH, Kim DH, Lim KH, et al. The impact of the hepatitis B virus polymerase rtA181T mutation on replication and drug resistance is potentially affected by overlapping changes in surface gene. J Virol. 2014;88:6805-18.

\section{Submit your next manuscript to BioMed Central and we will help you at every step:}

- We accept pre-submission inquiries

- Our selector tool helps you to find the most relevant journal

- We provide round the clock customer support

- Convenient online submission

- Thorough peer review

- Inclusion in PubMed and all major indexing services

- Maximum visibility for your research

Submit your manuscript at www.biomedcentral.com/submit
() Biomed Central 